\title{
Age Differences in Pet Sensitization by Pet Ownership
}

\author{
Jun Yeon Won ${ }^{1,2}$ [i] $\cdot$ Jea-Woo Kwon ${ }^{1,3}$ (i] $\cdot$ Seung-No Hong ${ }^{4}$ (i) $\cdot$ Woo Hyun Lee ${ }^{1}$ (i) \\ ${ }^{I}$ Department of Otolaryngology, Kangwon National University Hospital, Chuncheon; Departments of ${ }^{2}$ Otolaryngology and \\ ${ }^{3}$ Allergy and Clinical Immunology, Kangwon National University College of Medicine, Chuncheon; \\ ${ }^{4}$ Department of Otorhinolaryngology, SMG-SNU Boramae Medical Center, Seoul National University College of Medicine, Seoul, Korea
}

Objectives. The association between pet sensitization and pet ownership remains unclear. Therefore, we aimed to elucidate the association between pet sensitization and pet ownership by age.

Methods. We retrospectively reviewed 2,883 patients who visited our allergy clinic for nasal symptoms from January 2003 to December 2014, of whom 1,957 patients with data on skin-prick tests and questionnaire responses were included and divided into adults (age $>19$ years) and children (age $\leq 19$ years). The association between pet sensitization and pet ownership was evaluated in both groups.

Results. Among children, dog and cat sensitization showed no associations with dog and cat ownership, respectively. However, among adults, dog sensitization was significantly associated with dog ownership (odds ratio [OR], 3.283; $P<0.001$ ), and cat sensitization with cat ownership (OR, 13.732; $P<0.001)$. After adjustment for age, sex, familial history of allergy, sinusitis, diabetes mellitus, other pet ownership, and non-pet sensitization, significant associations remained between dog sensitization and dog ownership (adjusted OR [aOR], 3.881; $P<0.001$ ), and between cat sensitization and cat ownership (aOR, 10.804; $P<0.001$ ) among adults. Dog ownership did not show any association with allergic rhinitis, asthma, or atopic dermatitis, whereas atopic dermatitis had a significant association with cat ownership in adults (aOR, 4.840; $P<0.001)$.

Conclusion. Pet ownership in adulthood increased the risk of pet sensitization. However, pet ownership was not associated with the prevalence of atopic disorders, regardless of age, except for atopic dermatitis and cat ownership in adults.

Keywords. Pets; Ownership; Hypersensitivity; Allergic Rhinitis; Asthma; Atopic Dermatitis

\section{INTRODUCTION}

Atopic disorders, including allergic rhinitis (AR), asthma, and atopic dermatitis, are characterized by an immunoglobulin $\mathrm{E}$ (IgE)-mediated immune response that is triggered by an offending allergen [1]. Exposure of an atopic individual to indoor and outdoor allergens may contribute to the production of antigenspecific IgE, whereas allergen re-exposure can trigger early- and

- Received April 16, 2020

Revised July 15, 2020

Accepted August 14, 2020

- Corresponding author: Woo Hyun Lee

Department of Otolaryngology, Kangwon National University Hospital,

156 Baengnyeong-ro, Chuncheon 24289, Korea

Tel: +82-33-258-9109, Fax: +82-33-258-9486

E-mail: sniper212@hanmail.net late-stage reactions that lead to the clinical manifestations of atopic disorders [2].

Sensitization to furry animals, such as dogs and cats, is one of the main contributors to the development of atopic disorders [3]. Among atopic patients in the United States, pet dander is a common allergen, and the reported proportions of those with positive skin-prick tests (SPTs) to cat and dog dander were $72 \%$ and $36 \%$, respectively [4]. The prevalence of pet sensitization in childhood is relatively high in Korea, ranging from $4.9 \%$ to $11 \%$ [5]. Therefore, considering the high rate of pet sensitization in the general population and its effect on the development of atopic diseases, it is important to determine whether pet ownership has an impact on this condition. However, few studies have investigated the effect of pet ownership on the development of atopic diseases in adults, and studies of all age groups are scarce.

Copyright (c) 2021 by Korean Society of Otorhinolaryngology-Head and Neck Surgery.

This is an open-access article distributed under the terms of the Creative Commons Attribution Non-Commercial License (https://creativecommons.org/licenses/by-nc/4.0)

which permits unrestricted non-commercial use, distribution, and reproduction in any medium, provided the original work is properly cited. 
The association between pet ownership and pet sensitization has been investigated; however, the reported findings have been inconsistent and even conflicting. Moreover, there is no conclusive evidence on the relationship between keeping pets at home and developing atopic diseases [6]. For example, in a Dutch birth cohort study, the early presence of pets provided a protective effect against sensitization to inhaled allergens [7]. However, other studies have emphasized pet avoidance to prevent the development of atopic diseases in children with an atopic familial history $[8,9]$.

We postulated that there exists a difference between earlyand late-age clinical features associated with allergen sensitization and, therefore, that the relationship between pet ownership and sensitization would be different for children and adults. This study was conducted to investigate differences by age in pet sensitization and the prevalence of allergic diseases according to pet ownership.

\section{MATERIALS AND METHODS}

\section{Subjects}

This study was reviewed and approved by the Ethics Committee of Kangwon National University Hospital (IRB No. KNUH2019-04018) and written informed consent was exempted by the Institutional Review Board.

We retrospectively reviewed the result of SPT and a questionnaire survey of 2,883 patients who visited our allergy clinic from January 2003 to December 2014. We excluded 926 patients for the following reasons: negative result for histamine (positive control, $\mathrm{n}=3$ ) on SPT; positive result for saline (negative control, $n=11$ ) on SPT; the previous data of patients who underwent SPT twice ( $n=27)$; unavailable SPT result $(n=64)$; nonresponse to questionnaires $(n=814)$, and missing data on pet ownership $(\mathrm{n}=7)$. The final analysis dataset included 1,957 patients who completed questionnaires and had SPT results available.

\section{Skin-prick test}

A positive control (1.7 mg/mL histamine dihydrochloride), a negative control (0.9\% saline), and 11 allergens (Dermatophagoides farinae [Df], Dermatophagoides pteronyssinus [Dp], mug-

\section{H I G G H L L I}

- The relationship between pet ownership and pet sensitization is controversial.

- We identified differences in pet sensitization and the prevalence of atopic disorders according to age.

- For adult patients who own pets, clinicians should consider the associated risks of pet sensitization and atopic dermatitis. wort, ragweed, a grass pollen mixture [barley, oat, velvet grass, orchard grass, ryegrass, rye, wheat, timothy grass, Kentucky blue grass, and meadow fescue], Alternaria tenuis, Aspergillus fumigatus, dog epithelia, cat epithelia, a tree pollen mixture [alder, hazel, poplar, elm, and willow], and cockroaches) were dropped onto the volar surface of the patient's forearm, with 4-cm gap (approximately) between each drop. The results of the SPT was read 15 minutes after the allergen contact, and were considered positive when the mean wheal diameter for that allergen scored $3+$ (greater than or equal to that of the histamine control).

\section{Questionnaires and definition of atopic disorders}

Nasal symptoms in the preceding 12 months, without a cold, were evaluated using a 4-point Likert scale from 0 to $3(0$, no symptom; 1 , mild, 2 ; moderate; 3 , severe). The nasal symptom score was determined by points in every patient for each symptom such as nasal obstruction, nasal itching, sneezing, and rhinorrhea. Participants who recorded at least one nasal symptom score of more than 1 point were considered to have rhinitis.

Using a questionnaire, we investigated whether the patient was currently raising a pet at home, and if so, what kind of animal it was. The questionnaire gathered information on whether the parents of participants had problems with atopic disorders, such as AR, atopic dermatitis, and/or asthma, and participants with a positive response were considered to have a familial history of atopy. AR was defined as at least one or more positive responses to SPT with rhinitis. Non-allergic rhinitis (NAR) was defined as a negative response to SPT with rhinitis. Participants with a positive response to dog epithelia or cat epithelia were included in the pet-sensitized group, whereas participants with a positive response to other allergen, except pet allergen, were included in the non-pet-sensitized group. Asthma or atopic dermatitis was defined as "yes" in response to the question of "Have you ever been diagnosed with asthma (or atopic dermatitis) by doctor?" Moreover, the participant's medical history was recorded through the questionnaire.

\section{Statistical analysis}

We used SPSS ver. 18 (SPSS Inc., Chicago, IL, USA) to undertake data processing and analysis. All of the continuous variables are expressed as means and standard deviation. Independent $t$-tests and chi-square tests were used to compare the differences in continuous and categorical variable, respectively. Logistic regression analyses were conducted to evaluate the association between pet ownership and sensitization to pets (or development of atopic disorders). Crude odd ratios (OR) and their 95\% confidence intervals (CIs) were calculated. Multiple logistic regression models were created and included variables, such as general characteristics, past medical history, other pet ownership, and non-pet sensitization, of participants to estimate their adjusted OR (aOR) and $95 \%$ CI. A $P$-value $<0.05$ was considered significant. 


\section{RESULTS}

\section{General characteristics according to sensitization}

The sex distribution in this study population was $57.5 \%$ males $(n=1,126)$ and $42.5 \%$ females $(n=831)$, and the mean age of the participants was $41.2 \pm 21.1$ years. There were several differences between the AR and NAR groups with regard to participants' characteristics. The AR group was significantly younger and had a longer symptom duration than the NAR group $(P<$ $0.001)$. The proportion of males and familial history of atopy were higher in the AR group than in the NAR group $(P=0.018)$. However, no significant difference was found with regard to pet ownership between the AR and NAR groups $(P=0.139)$. With regard to past medical history, asthma $(P<0.001)$ and atopic dermatitis $(P=0.001)$ were more prevalent in the AR group than in the NAR group, whereas diabetes mellitus was more prevalent in the NAR group than in the AR group $(P<0.001)$. All nasal symptom scores, including nasal obstruction, rhinorrhea, sneezing, and itching, were significantly higher in the AR group than in the NAR group $(P<0.001$ for each). Differences in participant characteristics were evaluated between pet allergen-sensitized and other allergen-sensitized participants. The pet-sensitized group showed a significantly higher rate of pet ownership $(P<$ $0.001)$ and longer duration of nasal symptoms $(P=0.003)$ than the non-pet-sensitized group. However, nasal symptom scores were comparable between the groups. There were no betweengroup differences with regard to past medical history or disease prevalence, except that atopic dermatitis was more prevalent in the pet-sensitized group $(P=0.027)$ (Table 1$)$.

The distribution of offending allergens among participants is presented in Fig. 1. Sensitization to Df and Dp was the most common, followed by mugwort, tree pollen mixture, and ragweed. Sensitization to cat and dog dander was relatively low, with rates of $5.6 \%(n=110)$ and $2.4 \%(n=48)$, respectively. During the study period, dog ownership $(n=318,16.2 \%)$ was the most common type of pet ownership, followed by ownership of cats $(n=49$, $2.5 \%$ ), hamsters (0.5\%), and rabbits (0.3\%) (Fig. 2).

\section{Pet sensitization and atopic disorders according to pet ownership}

Asthma and AR were not related to pet ownership. However, atopic dermatitis was significantly associated with cat ownership; $31.1 \%$ of participants with cats had atopic dermatitis, whereas $10.4 \%$ of participants without cats had atopic dermatitis $(P<$ 0.001) (Table 2).

Pet sensitization was significantly associated with pet ownership. The estimated ORs of dog sensitization for dog ownership and cat sensitization for cat ownership were 3.452 (95\% CI, 1.885-6.321) and 9.763 (95\% CI, 5.173-18.429), respectively.

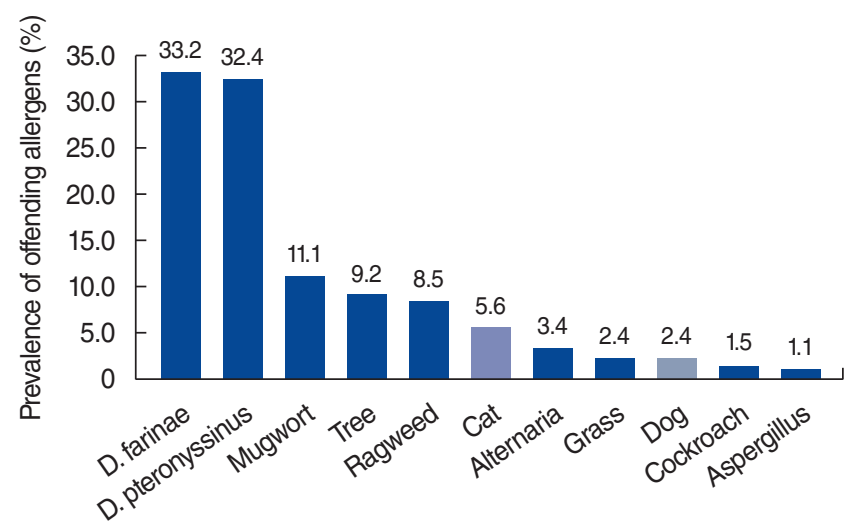

Fig. 1. The prevalence of offending allergens between 2003 and 2014. During this period, Dermatophagoides (D.) farina sensitization and $D$. pteronyssinus sensitization were the most common, whereas cat and dog sensitization rates were $5.6 \%$ and $2.4 \%$, respectively.

Table 1. Participants' characteristics according to allergen sensitization

\begin{tabular}{lcccc}
\hline Characteristics & All $(n=1,957)$ & Pet sensitized $(n=124)$ & Non-pet sensitized $(n=786)$ & Non-allergic rhinitis $(n=1,047)$ \\
\hline Age $(\mathrm{yr})$ & $41.22 \pm 21.1$ & $33.1 \pm 15.2$ & $34.9 \pm 18.4$ & $47.0 \pm 21.9$ \\
Male sex (\%) & $1,126(57.5)$ & $74(59.7)$ & $473(60.2)$ & $579(55.3)$ \\
Symptom duration (mo) & $55.6 \pm 70.1$ & $78.6 \pm 66.0$ & $59.5 \pm 67.2$ & $49.9 \pm 72.1$ \\
Atopy, familial history (\%) & $414(21.2)$ & $35(28.2)$ & $207(26.3)$ & $172(16.4)$ \\
Pet ownership & $385(19.7)$ & $44(35.5)$ & $125(15.9)$ & $216(20.6)$ \\
Past medical history & & & & \\
$\quad$ Asthma (\%) & $115(5.9)$ & $10(8.1)$ & $62(7.9)$ & $43(4.1)$ \\
Sinusitis (\%) & $285(14.6)$ & $13(10.5)$ & $110(14.0)$ & $162(15.5)$ \\
Atopic dermatitis (\%) & $213(10.9)$ & $24(16.5)$ & $19(2.4)$ & $92(8.8)$ \\
Diabetes mellitus (\%) & $88(4.5)$ & $2(1.6)$ & & $67(6.4)$ \\
Nasal symptom score & & & $1.5 \pm 1.1$ & $1.1 \pm 1.0$ \\
$\quad$ Nasal obstruction & $1.3 \pm 1.1$ & $1.7 \pm 1.1$ & $1.9 \pm 1.0$ & $1.7 \pm 1.0$ \\
Rhinorrhea & $1.8 \pm 1.0$ & $2.0 \pm 0.9$ & $1.8 \pm 0.9$ & $1.6 \pm 1.1$ \\
Sneezing & $1.7 \pm 1.0$ & $2.0 \pm 0.9$ & $1.3 \pm 1.0$ & $0.8 \pm 1.0$ \\
Itching & $1.1 \pm 1.1$ & $1.3 \pm 1.1$ & & \\
\hline
\end{tabular}

Values are presented as mean \pm standard deviation or number (\%). 
The OR of pet sensitization remained significant after adjustment for age, sex, familial history of atopy, sinusitis, diabetes mellitus, other pet ownership, and non-pet sensitization (aOR, 3.547; $P<0.001$ for dog ownership and aOR, 10.886; $P<0.001$ for cat ownership). However, in the subgroup analysis, children and

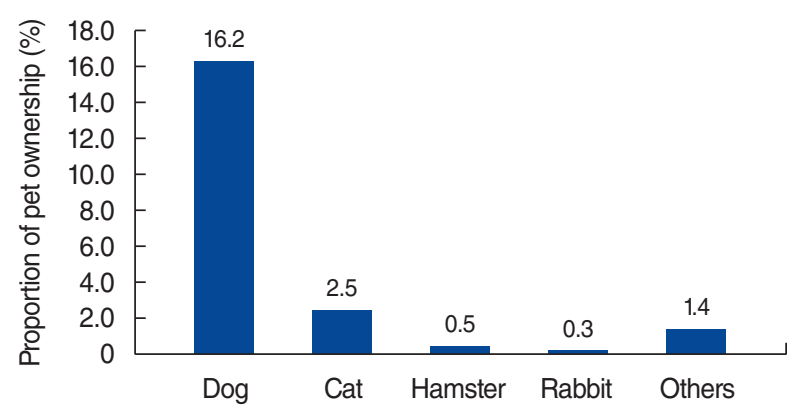

Fig. 2. The proportions of household pet ownership of participants. Dog ownership was the most common, followed by cat, hamster, and rabbit ownership. adults showed different results. In adults, dog and cat ownership was associated with a significantly elevated OR of dog and cat sensitization, respectively (aOR, 3.396; 95\% CI, 1.769-6.519 for dog ownership and aOR, 12.363; 95\% CI, 5.435-28.122 for cat ownership). However, among children, pet ownership was unrelated to pet sensitization.

Furthermore, the association between pet ownership and atopic disorders differed by age. AR was not associated with pet ownership in any age group. Dog ownership was unassociated with asthma, and none of the participants with cat ownership reported asthma. Dog ownership showed no protective or harmful effect on the presence of atopic dermatitis, regardless of age group. However, atopic dermatitis was associated with cat ownership, with different effects in children and adults. Cat ownership was significantly associated with atopic dermatitis in adults (aOR, 4.717; 95\% CI, 2.130-10.446), whereas it did not show any association among children (aOR, 0.752; 95\% CI, 0.148-3.819) (Table 3).

Table 2. Associations between pet ownership and atopic disorders

\begin{tabular}{|c|c|c|c|c|c|c|}
\hline \multirow{2}{*}{ Variable } & \multicolumn{3}{|c|}{ Dog ownership } & \multicolumn{3}{|c|}{ Cat ownership } \\
\hline & Yes & No & $P$-value & Yes & No & $P$-value \\
\hline Allergic rhinitis & $134(42.1)$ & 765 (46.7) & 0.137 & $25(52.1)$ & $874(45.8)$ & 0.387 \\
\hline Asthma & $18(5.7)$ & $97(5.9)$ & 0.858 & 0 & $115(6.0)$ & 0.080 \\
\hline Atopic dermatitis & $33(10.4)$ & $180(11.0)$ & 0.751 & 15 (31.3) & $198(10.4)$ & $<0.001^{*}$ \\
\hline
\end{tabular}

Values are presented as number (\%).

${ }^{\star} P<0.05$.

Table 3. Odds ratios for associations between pet ownership and sensitization to pet allergens

\begin{tabular}{|c|c|c|c|c|}
\hline \multirow{2}{*}{ Variable } & \multicolumn{2}{|c|}{ Dog ownership } & \multicolumn{2}{|c|}{ Cat ownership } \\
\hline & OR $(95 \% \mathrm{Cl})$ & Adjusted OR $(95 \% \mathrm{Cl})^{\mathrm{a})}$ & $\mathrm{OR}(95 \% \mathrm{Cl})$ & Adjusted OR $(95 \% \mathrm{Cl})^{\mathrm{a}}$ \\
\hline \multicolumn{5}{|l|}{ All $(n=1,957)$} \\
\hline Dog sensitization & $3.452(1.885-6.321)^{\star *}$ & $3.547(1.925-6.539)^{\star *}$ & & \\
\hline Cat sensitization & & & $9.763(5.173-18.429)^{\star \star}$ & $10.886(5.303-22.349)^{\star \star}$ \\
\hline Allergic rhinitis & $0.835(0.655-1.064)$ & $0.916(0.709-1.182)$ & $1.258(0.709-2.231)$ & $1.066(0.576-1.974)$ \\
\hline Asthma & $0.954(0.568-1.601)$ & $0.989(0.586-1.668)$ & NA & NA \\
\hline Atopic dermatitis & $0.939(0.634-1.389)$ & $1.052(0.698-1.586)$ & $3.928(2.097-7.359)^{\star *}$ & $2.971(1.484-5.947)^{\star}$ \\
\hline \multicolumn{5}{|c|}{ Children (age $\leq 19 \mathrm{yr}, \mathrm{n}=353$ ) } \\
\hline Dog sensitization & $4.489(0.730-27.605)$ & $4.455(0.632-31.411)$ & & \\
\hline Cat sensitization & & & $1.621(0.196-13.381)$ & $1.419(0.154-13.093)$ \\
\hline Allergic rhinitis & $0.698(0.378-1.292)$ & $0.657(0.349-1.238)$ & $0.318(0.081-1.249)$ & $0.307(0.074-1.276)$ \\
\hline Asthma & $0.677(0.197-2.322)$ & $0.613(0.177-2.132)$ & NA & NA \\
\hline Atopic dermatitis & $0.925(0.449-1.906)$ & $0.987(0.473-2.063)$ & $0.759(0.158-3.643)$ & $0.752(0.148-3.819)$ \\
\hline \multicolumn{5}{|c|}{ Adults (age $>19 \mathrm{yr}, \mathrm{n}=1,604$ ) } \\
\hline Dog sensitization & $3.283(1.728-6.236)^{\star *}$ & $3.396(1.769-6.519)^{\star *}$ & & \\
\hline Cat sensitization & & & $13.732(6.869-27.452)^{\star \star}$ & $12.363(5.435-28.122)^{\star \star}$ \\
\hline Allergic rhinitis & $0.882(0.677-1.149)$ & $0.953(0.718-1.266)$ & $1.755(0.915-3.367)$ & $1.190(0.580-2.442)$ \\
\hline Asthma & $1.073(0.604-1.906)$ & $1.119(0.624-2.008)$ & NA & NA \\
\hline Atopic dermatitis & $1.044(0.646-1.687)$ & $1.087(0.661-1.787)$ & $6.686(3.330-13.424)^{\star \star}$ & $4.717(2.130-10.446)^{*}$ \\
\hline
\end{tabular}

OR, odds ratio; $\mathrm{Cl}$, confidence interval; $\mathrm{NA}$, not applicable.

a)Adjusted for age, sex, familial history of atopy, sinusitis, diabetes mellitus, other pet ownership, and non-pet sensitization.

${ }^{\star} P<0.05,{ }^{*} P<0.001$. 


\section{DISCUSSION}

In this study, we investigated the following questions related to pet ownership: (1) Is there a difference between children and adults in pet sensitization according to pet ownership? and (2) Is there a difference between children and adults in the development of atopic disorders according to pet ownership? In adults, dog and cat ownership was significantly associated with dog and cat sensitization, respectively, although this association was not found in children. Furthermore, the only association found for any of the atopic disorders was an association between atopic dermatitis and cat ownership in adults.

Numerous previous studies have revealed associations between pet ownership and sensitization to pets; however, most of those studies were conducted in certain age groups. Previous studies have been performed on birth cohorts [10-13], school-age children $[14,15]$, and adults $[16,17]$. One strength of this study is that it was conducted across all age groups in a large populationbased survey of the community, and differences were found in the relationship between pet ownership and sensitization in children and adults.

Pet ownership can affect exposure and sensitization to pet allergens, and the frequency of pet ownership varies according to culture. Our study participants had a lower rate of pet ownership $(18.7 \%)$, including dogs and cats, than has been reported in the United States (45.1\%) [18]. Moreover, cat ownership was substantially less frequent in our study $(2.5 \%)$ than has been reported in Europe $(10 \%-50 \%)$ [19]. This study is unique because it showed a pattern of pet sensitization in a region with significantly lower exposure to community pet allergens. Moreover, most large population-based studies related to pet allergies have been conducted in Western countries and have been rarely undertaken in Asians, who have different ethnic characteristics $[16,20]$.

Recently, there has been increasing evidence that exposure to furry animals, such as dogs and cats, has a protective effect on allergic sensitization in children [21-24], but there have been many reports of harmful effects on allergic sensitization in adults $[16,25,26]$. Our findings showing that pet ownership had no effect on pet sensitization in children, but increased the risk of sensitization in adults, are partly consistent with previous findings. Given previous findings and our findings, it appears that late exposure to pets could increase sensitization and that adults should therefore avoid pet ownership. The discrepancy in pet sensitization by pet exposure between children and adults may be explained by the modified Th2 response, which occurs when a person exposed to high levels of antigens in childhood produces an immune response to control those antigens, such as IgG4 antibody release [27]. The relationship between pet exposure and the development of atopic disorders is controversial. Even in meta-analyses conducted to determine whether pet exposure has a protective or harmful effect on the development of atopic disorders, inconsistent results have been reported [28-30].
In a previous meta-analysis, cat exposure had protective effects on asthma, whereas dog exposure increased the risk of asthma. Furthermore, exposure to both pets appeared to have a protective effect on rhinitis [30]. However, this study did not find any associations between pet ownership and AR (or asthma), regardless of age group. As sensitization to allergen increases, we theorized that the prevalence of AR or asthma would increase. However, pet sensitization was increased by pet ownership in adults, whereas the prevalence of AR or asthma did not increase. This means that pet sensitization did not lead to the development of AR or asthma.

In general, it has been believed that pet exposure in childhood may help prevent the development of atopic dermatitis [31]; however, few studies have investigated the effects of pet exposure on atopic dermatitis in adults. To the best of our knowledge, this study is the first to find an association between cat ownership and atopic dermatitis in adults. The finding of no association between atopic dermatitis and pet ownership in children differed from those of a previous study reporting that cat ownership had the effect of preventing atopic dermatitis [29]. This discrepancy may be caused by differences in study design and/or population characteristics, such as age, racial differences, and the prevalence of cats in the community. Moreover, the difference between dog and cat ownership in the effect on atopic dermatitis may explain the difference in the biochemical characteristics of allergens. In brief, Canis familiaris allergen 1, the major dog allergen, is a lipocalin, whereas Felis domesticus allergen 4, the major cat allergen, is a secretoglobin [32,33]. Atopic disorders such as asthma, AR, and atopic dermatitis often coexist and are considered to be part of the same disease spectrum because they have the same risk factors and pathophysiology. However, in our study, pet ownership was only associated with atopic dermatitis in adults. Therefore, further research is needed on the details of the pathophysiology by which pet allergens cause the development of each atopic disease.

This study has several limitations. First, this study utilized a cross-sectional design and could not investigate whether the development of atopic disorders was affected by the duration of pet ownership. Moreover, it is possible that many of the adults in this study had owned pets since childhood.Therefore, a causal relationship between atopic disorders and pet ownership could not be confirmed. Second, the study was conducted among participants who visited a doctor. This may have caused selection bias, because participants with more severe symptoms are more likely to seek medical care for their condition. The participants in this study may have had more atopic disorders and a higher rate of pet sensitization than the general population. Furthermore, our study results may not apply to the general population. Third, it is well known that socioeconomic factors such as smoking, education, housing, and income are important factors implicated in the development of atopic disorders. However, this study could not obtain information on socioeconomic factors through 
the questionnaires. Therefore, well-designed follow-up studies are needed to confirm possible age-specific associations between pet ownership and pet sensitization. Fourth, there are differences in the diagnostic criteria of asthma and atopic dermatitis. In this study, asthma and atopic dermatitis were defined by simple questions that asked whether the participant had been diagnosed with asthma or atopic dermatitis by a doctor. There is no consensus on the definition of asthma [34], and atopic dermatitis is often diagnosed by typical clinical features [35]. Therefore, some researchers may disagree with our definitions of asthma and atopic dermatitis, although we used limited questionnaire data to define the most reasonable diagnosis.

In conclusion, differences were identified between children and adults regarding the association between pet ownership and sensitization to pets. Having a pet in adulthood increased the risk of pet sensitization. However, pet ownership was not associated with the presence of atopic disorders regardless of age, with the exception of an association between atopic dermatitis and cat ownership in adults.

\section{CONFLICT OF INTEREST}

No potential conflict of interest relevant to this article was reported.

\section{ACKNOWLEDGMENTS}

This research was supported by the Basic Science Research Program through the National Research Foundation of Korea (NRF) funded by the Ministry of Education (2019R1F1A1051106).

We would like to express our sincerest gratitude to Hyun Kyung Choi, who worked in the allergy clinic.

\section{ORCID}

Jun Yeon Won $\quad$ https://orcid.org/0000-0002-1615-3760

Jea-Woo Kwon https://orcid.org/0000-0003-1639-3606

Seung-No Hong https://orcid.org/0000-0002-5759-9929

Woo Hyun Lee https://orcid.org/0000-0001-7725-4146

\section{AUTHOR CONTRIBUTIONS}

Conceptualization: JYW, WHL. Data curation: JYW, JWK. Formal analysis: WHL, SNH. Funding acquisition:WHL. Methodology: JYW, WHL. Project administration: WHL. Visualization: JWK, SNH. Writing-original draft: JYW, WHL. Writing-review \& editing: SNH,WHL.

\section{REFERENCES}

1. Bousquet J, Khaltaev N, Cruz AA, Denburg J, Fokkens WJ, Togias A, et al.Allergic Rhinitis and its Impact on Asthma (ARIA) 2008 update (in collaboration with the World Health Organization, GA(2)LEN and AllerGen). Allergy. 2008 Apr;63 Suppl 86:8-160.

2. Vinke JG, KleinJan A, Severijnen LW, Hoeve LJ, Fokkens WJ. Differences in nasal cellular infiltrates between allergic children and agematched controls. Eur Respir J. 1999 Apr;13(4):797-803.

3. Perzanowski MS, Ronmark E, Platts-Mills TA, Lundback B. Effect of cat and dog ownership on sensitization and development of asthma among preteenage children. Am J Respir Crit Care Med. 2002 Sep; 166(5):696-702.

4. Craig TJ, King TS, Lemanske RF Jr,Wechsler ME, Icitovic N, Zimmerman RR Jr, et al. Aeroallergen sensitization correlates with PC(20) and exhaled nitric oxide in subjects with mild-to-moderate asthma. J Allergy Clin Immunol. 2008 Mar;121(3):671-7.

5. Kim DH, Park YS, Jang HJ, Kim JH, Lim DH. Prevalence and allergen of allergic rhinitis in Korean children. Am J Rhinol Allergy. 2016 May; 30(3):72-8.

6. Salo PM, Zeldin DC. Does exposure to cats and dogs decrease the risk of allergic sensitization and disease? J Allergy Clin Immunol. 2009 Oct;124(4):751-2.

7. Kerkhof M, Wijga AH, Brunekreef B, Smit HA, de Jongste JC, Aalberse RC, et al. Effects of pets on asthma development up to 8 years of age: the PIAMA study. Allergy. 2009 Aug;64(8):1202-8.

8. Almqvist C, Egmar AC, van Hage-Hamsten M, Berglind N, Pershagen G, Nordvall SL, et al. Heredity, pet ownership, and confounding control in a population-based birth cohort. J Allergy Clin Immunol. 2003 Apr;111(4):800-6.

9. Bornehag CG, Sundell J, Hagerhed L, Janson S; DBH Study Group. Pet-keeping in early childhood and airway, nose and skin symptoms later in life.Allergy. 2003 Sep;58(9):939-44.

10. Arshad SH, Tariq SM, Matthews S, Hakim E. Sensitization to common allergens and its association with allergic disorders at age 4 years: a whole population birth cohort study. Pediatrics. 2001 Aug; 108(2):E33.

11. Lindfors A, van Hage-Hamsten M, Rietz H, Wickman M, Nordvall SL. Influence of interaction of environmental risk factors and sensitization in young asthmatic children. J Allergy Clin Immunol. 1999 Oct;104(4 Pt 1):755-62.

12. Munir AK, Kjellman NI, Bjorksten B. Exposure to indoor allergens in early infancy and sensitization. J Allergy Clin Immunol. 1997 Aug; 100(2):177-81.

13. Ownby DR, Johnson CC. Does exposure to dogs and cats in the first year of life influence the development of allergic sensitization? Curr Opin Allergy Clin Immunol. 2003 Dec;3(6):517-22.

14. Hesselmar B, Aberg N, Aberg B, Eriksson B, Bjorksten B. Does early exposure to cat or dog protect against later allergy development? Clin Exp Allergy. 1999 May;29(5):611-7.

15. Ritz BR, Hoelscher B, Frye C, Meyer I, Heinrich J. Allergic sensitization owing to 'second-hand' cat exposure in schools. Allergy. 2002 Apr;57(4):357-61.

16. Park YB, Mo EK, Lee JY, Kim JH, Kim CH, Hyun IG, et al. Association between pet ownership and the sensitization to pet allergens in adults with various allergic diseases. Allergy Asthma Immunol Res. 2013 Sep;5(5):295-300.

17. Roost HP, Kunzli N, Schindler C, Jarvis D, Chinn S, Perruchoud AP, et al. Role of current and childhood exposure to cat and atopic sensitization. European Community Respiratory Health Survey. J Allergy Clin Immunol. 1999 Nov;104(5):941-7.

18. Arbes SJ Jr, Cohn RD, Yin M, Muilenberg ML, Friedman W, Zeldin DC. Dog allergen (Can f 1) and cat allergen (Fel d 1) in US homes: 
results from the National Survey of Lead and Allergens in Housing J Allergy Clin Immunol. 2004 Jul;114(1):111-7.

19. Heinrich J, Bedada GB, Zock JP, Chinn S, Norback D, Olivieri M, et al. Cat allergen level: its determinants and relationship to specific IgE to cat across European centers. J Allergy Clin Immunol. 2006 Sep; 118(3):674-81.

20. Zheng T, Niu S, Lu B, Fan X, Sun F, Wang J, et al. Childhood asthma in Beijing, China: a population-based case-control study. Am J Epidemiol. 2002 Nov;156(10):977-83.

21. Custovic A, Hallam CL, Simpson BM, Craven M, Simpson A, Woodcock A. Decreased prevalence of sensitization to cats with high exposure to cat allergen. J Allergy Clin Immunol. 2001 Oct;108(4): 537-9.

22. Custovic A, Simpson BM, Simpson A, Hallam CL, Marolia H, Walsh $\mathrm{D}$, et al. Current mite, cat, and dog allergen exposure, pet ownership, and sensitization to inhalant allergens in adults. J Allergy Clin Immunol. 2003 Feb;111(2):402-7.

23. Oryszczyn MP, Annesi-Maesano I, Charpin D, Kauffmann F. Allergy markers in adults in relation to the timing of pet exposure: the EGEA study. Allergy. 2003 Nov;58(11):1136-43.

24. Ownby DR, Johnson CC, Peterson EL. Exposure to dogs and cats in the first year of life and risk of allergic sensitization at 6 to 7 years of age. JAMA. 2002 Aug;288(8):963-72.

25. Linneberg A, Nielsen NH, Madsen F, Frolund L, Dirksen A, Jorgensen T. Pets in the home and the development of pet allergy in adulthood. The Copenhagen Allergy Study. Allergy. 2003 Jan;58(1):21-6.

26. Jaakkola JJ, Jaakkola N, Piipari R, Jaakkola MS. Pets, parental atopy, and asthma in adults. J Allergy Clin Immunol. 2002 May;109(5):
784-8.

27. Platts-Mills TA, Woodfolk JA, Erwin EA, Aalberse R. Mechanisms of tolerance to inhalant allergens: the relevance of a modified Th2 response to allergens from domestic animals. Springer Semin Immunopathol. $2004 \mathrm{Feb} ; 25(3-4): 271-9$.

28. Apelberg BJ, Aoki Y, Jaakkola JJ. Systematic review: exposure to pets and risk of asthma and asthma-like symptoms. J Allergy Clin Immunol. 2001 Mar;107(3):455-60.

29. Langan SM, Flohr C, Williams HC. The role of furry pets in eczema: a systematic review.Arch Dermatol. 2007 Dec;143(12):1570-7.

30. Takkouche B, Gonzalez-Barcala FJ, Etminan M, Fitzgerald M. Exposure to furry pets and the risk of asthma and allergic rhinitis: a metaanalysis. Allergy. 2008 Jul;63(7):857-64.

31. Pelucchi C, Galeone C, Bach JF, La Vecchia C, Chatenoud L. Pet exposure and risk of atopic dermatitis at the pediatric age: a meta-analysis of birth cohort studies. J Allergy Clin Immunol. 2013 Sep;132(3): 616-22.

32. Kaiser L, Gronlund H, Sandalova T, Ljunggren HG, van Hage-Hamsten M,Achour A, et al.The crystal structure of the major cat allergen Fel d 1, a member of the secretoglobin family. J Biol Chem. 2003 Sep;278(39):37730-5.

33. Virtanen T. Lipocalin allergens. Allergy. 2001;56 Suppl 67:48-51.

34. Mims JW. Asthma: definitions and pathophysiology. Int Forum Allergy Rhinol. 2015 Sep;5 Suppl 1:S2-6.

35. Eichenfield LF,Tom WL, Chamlin SL, Feldman SR, Hanifin JM, Simpson EL, et al. Guidelines of care for the management of atopic dermatitis: section 1: diagnosis and assessment of atopic dermatitis. J Am Acad Dermatol. 2014 Feb;70(2):338-51. 